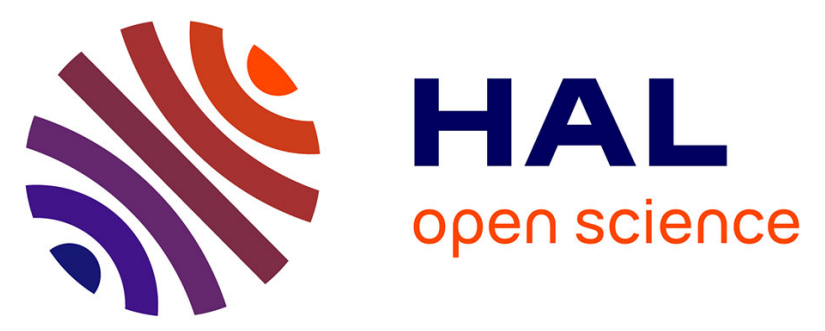

\title{
Beta Decay Studies of Neutron Rich Nuclei Using Total Absorption Gamma-ray Spectroscopy and Delayed Neutron Measurements
}

\author{
J.L. Tain, A. Algora, J. Äysto, L. Batist, M. Bowry, M. Bunce, R. Caballero, \\ D. Cano-Ott, G. Cortes, C. Domingo-Pardo, et al.
}

\section{To cite this version:}

J.L. Tain, A. Algora, J. Äysto, L. Batist, M. Bowry, et al.. Beta Decay Studies of Neutron Rich Nuclei Using Total Absorption Gamma-ray Spectroscopy and Delayed Neutron Measurements. International Conference on Nuclear Data for Science and Technology ND2010, Apr 2010, Jeju Island, South Korea. pp.1499-1502, 10.3938/jkps.59.1499 . in2p3-00686870

\section{HAL Id: in2p3-00686870 \\ https://hal.in2p3.fr/in2p3-00686870}

Submitted on 28 Jun 2021

HAL is a multi-disciplinary open access archive for the deposit and dissemination of scientific research documents, whether they are published or not. The documents may come from teaching and research institutions in France or abroad, or from public or private research centers.
L'archive ouverte pluridisciplinaire HAL, est destinée au dépôt et à la diffusion de documents scientifiques de niveau recherche, publiés ou non, émanant des établissements d'enseignement et de recherche français ou étrangers, des laboratoires publics ou privés.

\section{(c)(1)}

Distributed under a Creative Commons Attribution| 4.0 International License 


\title{
Beta Decay Studies of Neutron Rich Nuclei Using Total Absorption Gamma-ray Spectroscopy and Delayed Neutron Measurements
}

\author{
J. L. Tain, ${ }^{*}$ A. Algora, E. Estevez, B. Rubio, E. Valencia and D. Jordan \\ Instituto de Fisica Corpuscular, CSIC-University of Valencia, Valencia 46071, Spain \\ J. Äysto, T. Eronen, A. Jokinen, I. Moore, H. Pentillä and J. Riisanen \\ Department of Physics, University of Jyväskylä, Jyväskylä 40014, Finland \\ L. BATIST \\ Petersburg Nuclear Physics Institute, Gatchina 188300, Russia \\ M. Bowry, M. Bunce and W. Gelletly \\ Department of Physics, University of Surrey, Guilford, GU2 7XH, UK \\ R. Caballero, G. Cortes, B. Gomez-Hornillos and V. Gorlychev \\ Seccio d'Enginyeria Nuclear, Univesitat Politecnica de Catalunya, Barcelona 08028, Spain \\ D. Cano-Ott, A. Garcia and T. Martinez \\ Centro de Investigaciones Energeticas Medioambientales y Tecnologicas, Madrid 28040, Spain \\ C. Domingo-Pardo \\ GSI Helmholtzzentrum für Schwerionenforschung GmbH, Darmstadt 64291, Germany \\ M. Fallot, L. Giot, A. Porta and B. Van Vinh \\ Subatech laboratory, CNRS/IN2P3 - Université de Nantes, Nantes 44307, France
}

\begin{abstract}
A complete characterisation of the $\beta$-decay of neutron-rich nuclei can be obtained from the measurement of $\beta$-delayed gamma rays and, whenever the process is energetically possible, $\beta$-delayed neutrons. The accurate determination of the $\beta$-intensity distribution and the $\beta$-delayed neutron emission probability is of great relevance in the fields of reactor technology and nuclear astrophysics. A programme for combined measurements using the total absorption gamma-ray spectroscopy technique and both neutron counters and neutron time-of-flight spectrometers is presented.
\end{abstract}

\section{INTRODUCTION}

Neutron-rich nuclei are produced abundantly in nuclear reactors as a by-product of the neutron-induced fission of heavy elements present in the fuel assembly. They are also produced in the neutron capture processes which lead to the formation of the heavier elements in the Universe. Extremely neutron-rich nuclei are produced in the so-called rapid neutron capture process (or r-process) which takes place when elements around Fe

*E-mail: tain@ific.uv.es are subjected to an intense pulse of neutrons.

The $\beta$-unstable nuclei produced in this way decay with the emission of an electron and an antineutrino, often leaving the daughter nucleus in an excited state which releases its energy by $\gamma$-ray emission. As the neutron excess increases the neutron separation energy in the daughter nucleus $S_{n}$ moves below the accessible decay energy window $Q_{\beta}$ and the emission of one or more neutrons from states above the neutron separation energy competes efficiently with the electromagnetic deexcitation.

The distribution of $\beta$-decay intensity $I_{\beta}$ and the frac- 
tion proceeding through neutron emission $P_{n}$ are fundamental quantities which depend on the structure of parent and daughter nucleus. This information is contained in the $\beta$-strength distribution $S_{\beta}$ which measures the transition probability between initial and final states. The relation between intensity and strength depends also on the energy available to the decay (through the Fermi rate function $\left.f \sim\left(Q_{\beta}-E_{x}\right)^{5}\right)$ and the half-life $T_{1 / 2}$

$$
S_{\beta}\left(E_{x}\right)=\frac{I_{\beta}\left(E_{x}\right)}{f\left(Q_{\beta}-E_{x}\right) \cdot T_{1 / 2}} .
$$

As can be seen from eq. (1) the decay half-life represents a weighted average of $S_{\beta}$

$$
\frac{1}{T_{1 / 2}}=\int_{0}^{Q_{\beta}} S_{\beta}\left(E_{x}\right) \cdot f\left(Q_{\beta}-E_{x}\right) d E_{x} .
$$

On the other hand the weighted integral of the strength above $S_{n}$ determines the neutron emission probability

$$
P_{n}=\frac{\int_{S_{n}}^{Q_{\beta}} S_{\beta}\left(E_{x}\right) \cdot f\left(Q_{\beta}-E_{x}\right) \cdot \frac{\Gamma^{n}\left(E_{x}\right)}{\Gamma^{t o t}\left(E_{x}\right)} d E_{x}}{\int_{0}^{Q_{\beta}} S_{\beta}\left(E_{x}\right) \cdot f\left(Q_{\beta}-E_{x}\right) d E_{x}} .
$$

Here the competition between neutron emission and electromagnetic de-excitation is represented by the ratio of widths $\Gamma^{n} / \Gamma^{t o t}$.

The $P_{n}$ value determines the delayed neutron fraction in a reactor, a crucial ingredient of reactor kinetics, while the total decay pattern $I_{\beta}$ determines the reactor decay heat ( $\beta$ and $\gamma$ components) and the anti-neutrino spectrum released from the reactor. In the astrophysical context, the $S_{\beta}$ distribution determines the half-life of the nuclei along the production path in the rapid neutron capture process, shaping their abundance distribution and the overall speed of the process, while the delayed neutron emission probability $P_{n}$ influences the final elemental abundances.

A programme of combined measurements, using total absorption gamma-ray spectroscopy and both a $4 \pi$ neutron counter and a neutron time-of-flight spectrometer has been started, which is aimed at the determination of the complete $\beta$-decay distribution of relevant neutronrich nuclei. Initial measurements performed at JYFL (Jyväskylä) will be described and plans for the measurement of more exotic nuclei at future radioactive beam facilities will be outlined.

\section{MEASUREMENTS AT IGISOL (JYFL)}

The IGISOL facility [1] at the University of Jÿvaskÿla (Finland) is equipped with an ion guide type ion source which uses a He gas flow to carry the reaction products emerging from a thin target into the mass separator. In contrast with other methods this procedure is chemically insensitive thus allowing the study of refractory elements, which was one of our goals. Another key characteristic of the installation is the existence of a dual Penning trap system JYFLTRAP [2] which can be used as a mass separator with extreme resolution, which allows the separation of isobars or even isomers. This is essential when applying non-selective detection techniques, where any spectrum contamination affects the accuracy of the result. The combination of these two features makes IGISOL a unique facility for this type of measurement.

Measurements were performed during November 2009 on a series of neutron rich $\mathrm{Br}, \mathrm{Rb}$ and I isotopes using a Total Absorption Spectrometer (TAS) and a $4 \pi$ neutron counter. The isotopes were produced by proton and deuteron-induced fission in a Uranium target. The purified beam extracted from the trap was collimated and implanted directly onto a movable tape at the centre of the detector. This allows measurements on short lived species without loss of statistics. After a conveniently chosen measuring time the activity was removed by the tape and a new accumulation cycle started.

The nuclei investigated were chosen from the NEA/WPEC-25 priority list [3] of nuclei which are expected to contribute significantly to the solution of discrepancies between reactor decay heat integral measurements and summation calculations. Existing information in nuclear data bases on the decay pattern for these isotopes is likely to be affected by the systematic errors associated with the Pandemonium effect which distorts the decay intensity distribution towards low excitation energies [4]. Some of these nuclei are also expected to contribute to the discrepancies between measured and calculated anti-neutrino spectrum from reactors. It is important to improve on these measurements in the context of remote monitoring of proliferation activities in reactors and the analysis of reactor neutrino oscillation experiments [5]. A third motivation is related to the understanding of the evolution of nuclear structure in neutron-rich nuclei around the mass $\mathrm{A} \sim 100$ region where rapid shape changes are expected.

\section{Total Absorption $\gamma$-Ray Spectroscopy}

The measurements were performed with a new spectrometer composed of 12 independent $\mathrm{BaF}_{2}$ crystals arranged in a compact cylindrical geometry with minimum dead material in between. A detection efficiency close to $100 \%$ is attained for $\gamma$-ray cascades. An inner hole of $5 \mathrm{~cm}$ diameter allows the insertion of the vacuum beam tube containing the tape transport system. A $1 \mathrm{~mm}$ thick Si detector is placed inside the tube behind the tape implantation point. This allows tagging of the spectrometer signals with the coincident $\beta$-particles in order to obtain background-free spectra. A dedicated data ac- 


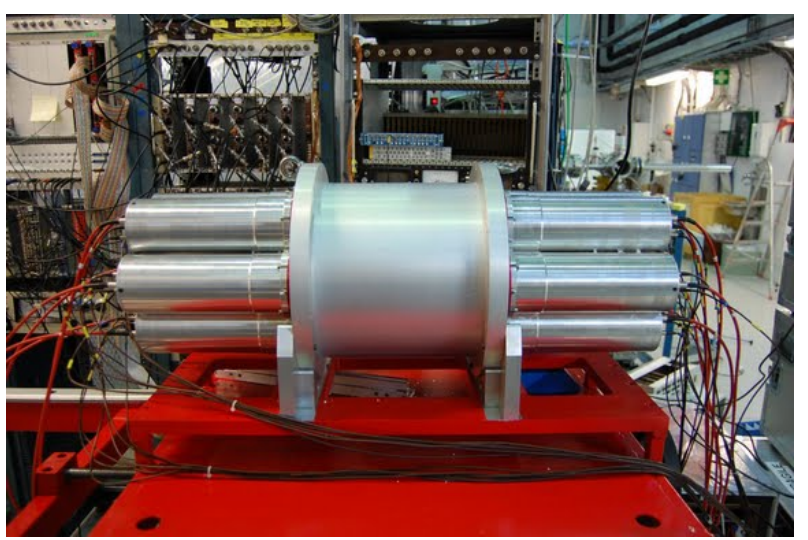

Fig. 1. (Color online) View of the total absorption spectrometer without lead shielding.

quisition system has been developed which allows online gain matching of the $12 \mathrm{PMT}$ signals through small HV adjustments. The reference signals for the matching are provided by the intrinsic $\alpha$-activity of the Ra contaminants in $\mathrm{BaF}_{2}$. Alpha peaks are isolated using the differences in the fast/slow component of the scintillation light pulse. The excellent timing properties of $\mathrm{BaF}_{2}$ can be used to discriminate against neutron-induced signals in the case of neutron emitters, which are slightly delayed in time.

Spectra with sufficient statistics were acquired for seven isotopes: ${ }^{86-88} \mathrm{Br},{ }^{91-94} \mathrm{Rb}$. Data were registered during the beam implantation time of about $2-3$ times the isotope half-life, before moving the tape. If the accumulated daughter activity was significant a dedicated measurement was performed to correct for its contribution to the spectrum. The on-line spectra revealed significant population of levels at high excitation energies and a rich and isotope dependent structure in the strength distribution. The analysis will be performed using the methods developed by the group in Valencia [6]. It is worth mentioning that this is the first time that a segmented TAS has been applied to $\beta$-decay studies allowing $\gamma$-ray cascade multiplicities to be measured. This information will be used to reduce further any dependence of the result on the unknown de-excitation path [7].

\section{Delayed Neutron Measurements}

Measurements were performed using the newly built BEta deLayEd Neutron (BELEN) detector. The detector consists of 20 high pressure, $60 \mathrm{~cm}$ long, ${ }^{3} \mathrm{He}$ proportional counters embedded in a polyethylene matrix which acts as a neutron moderator. A neutron detection efficiency of about 30\% (from Monte Carlo simulations) with small energy dependence could be obtained with an optimised distribution of the counters in two rings around the central hole. A more detailed description of the detector can be found in [8]. Apart from the beam tube the central hole accommodated a large volume HPGe detector which was used for identification
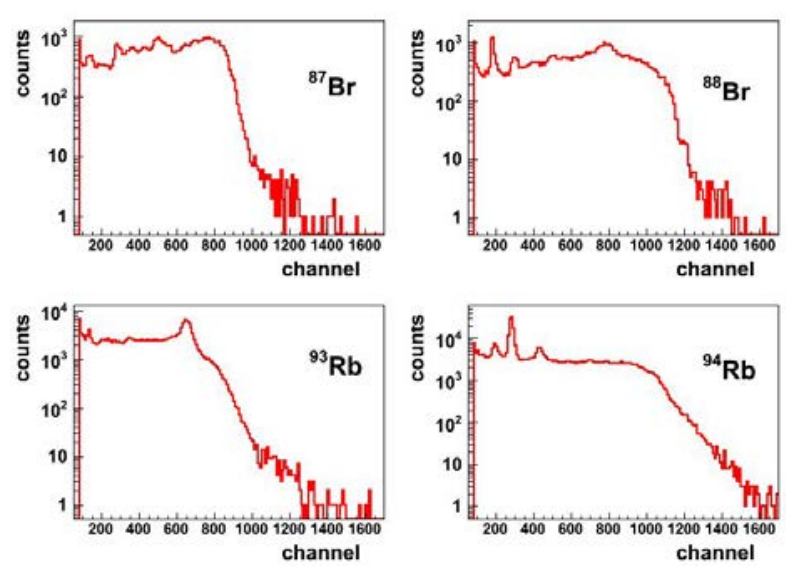

Fig. 2. (Color online) Some $\beta$-gated TAS spectra recorded on-line.

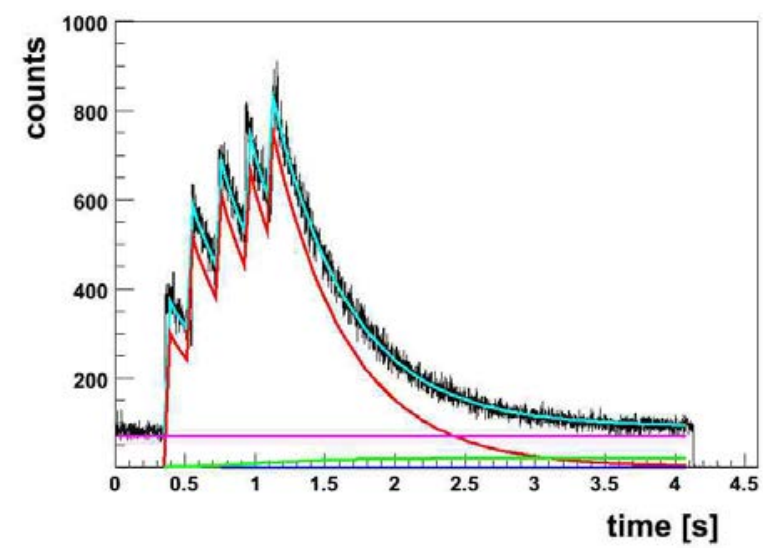

Fig. 3. (Color online) Time distribution of ${ }^{95} \mathrm{Rb} \beta$-signals and its decomposition using the Bateman equations. The time structure of the beam released from the Penning trap is clearly visible.

of the accumulated activity. A new trigger-less DACQ system based on sampling digitizers was developed for these measurements. This system provides independent time-stamped energy information for each counter allowing event-by-event optimal noise rejection and millisecond time correlations with a minimum dead time. Additional polyethylene shielding was added in order to reduce the level of detected neutron background signals to about $0.8-1.5 \mathrm{n} / \mathrm{s}$.

In this initial measurement data were accumulated for the well known neutron emitters ${ }^{88} \mathrm{Br},{ }^{94,95} \mathrm{Rb}$ and ${ }^{138} \mathrm{I}$, in order to verify the setup and calibrate the neutron detection efficiency $\varepsilon_{\mathrm{n}}$. The beam was implanted onto the tape at the centre of the detector for a period equivalent to $3 \times \mathrm{T}_{1 / 2}$, while the measuring time lasted up to 10 $\times T_{1 / 2}$. The resulting growth and decay activity curves in the $\beta$ and neutron detectors can be decomposed into the activities of the parent and its progeny using the Bateman equations. The parent $\beta$-activity $N_{\beta}$ and the correlated $\beta$-neutron activity $N_{\beta n}$ can then be used to obtain $P_{n}$ (or conversely $\varepsilon_{\mathrm{n}}$ for well know $P_{n}$ ) according 


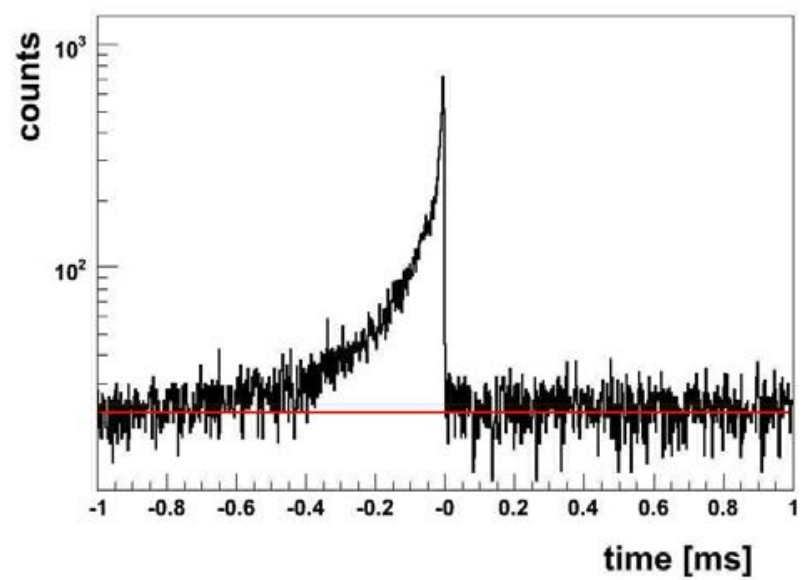

Fig. 4. (Color online) Time correlation between a ${ }^{95} \mathrm{Rb}$ neutron signal and all $\beta$-signals in the shown time interval. The distribution reflects the neutron moderation plus capture time.

to the relation

$$
P_{n}=\frac{1}{\varepsilon_{n}} \frac{N_{\beta n}}{N_{\beta}} .
$$

The data analysis is still in progress.

\section{FUTURE PLANS}

New experiments will be performed at JYFL after IGISOL is moved to its new location [9]. The goal will be to study fission products with larger neutron excess. Measurements will be made using both the TAS and BELEN detectors. Additional measurements will be performed with a time-of-flight neutron spectrometer currently under development. The spectrometer will consist of 30 BC501A organic liquid scintillation cells supplemented with a fast plastic scintillation $\beta$-detector and HPGe detectors. Dedicated fast sampling digitizer cards are being developed for optimum neutron/gamma separation. The measured neutron energy spectra will allow the reconstruction of the strength distribution above the neutron separation energy $S_{n}$.

The study of even more neutron-rich nuclei will require the use of advanced methods of production and detection. This will be the case for the Facility for Antiproton and Ion Research (FAIR) being built at Darmstadt (Germany). Within the DESPEC experiment [10] of the FAIR/NUSTAR collaboration, measurements of the decay properties of very neutron-rich nuclei along the lines presented above are planned. Similar experiments with much lower intensities can be performed at the present GSI (Darmstadt) facility. A proposal has been presented recently to investigate the decay properties of several Ir -Tl isotopes [11]. These isotopes will be produced by high energy fragmentation of a $1 \mathrm{GeV} / \mathrm{u}{ }^{238} \mathrm{U}$ beam. The high velocity fragments will be selected according to their $B \rho$ value in the Fragment Separator (FRS) and implanted into a stack of DSSSD. Every implanted isotope will be identified combining the information of its time-of-flight through the FRS and energy loss within an ionization chamber at the exit of the FRS. For each pixel of the DSSSD the time correlation between the implantation signal and the decay signal will provide $T_{1 / 2}$ and the correlation with the signals in the BELEN neutron detector will provide $P_{n}$. Even if the very limited statistics prevent a TAS measurement, gross information on the $S_{\beta}$ distribution will be gained since according to Eqs. (2) and (3), $T_{1 / 2}$ is sensitive to the strength at low excitation energy and $P_{n}$ is sensitive to the strength at high excitation energy. This will help to refine the theoretical models required to predict the decay properties of r-process nuclei in the vicinity of the so called $3^{\text {rd }}$ abundance peak addressed in the present case.

\section{ACKNOWLEDGMENTS}

This work was partially supported by Spanish FPA2008-06419-C02-01 grant.

\section{REFERENCES}

[1] J. Ärje et al., Nucl. Instrum. Methods Phys. Res. Sect. A 247, 431 (1986).

[2] V. S. Kolhinen et al., Nucl. Instrum. Methods Phys. Res. Sect. A 528, 776 (2004).

[3] T. Yoshida et al., Working Party on International Evaluation Co-operation Subgroup 25, NEA/OECD No 6284, 2007.

[4] A. Algora et al., Eur. Phys. J-Spec. Top. 150, 383 (2007).

[5] M. Fallot et al., in Proceedings of Inter. Conf. on Nucl. Data for Sci. and Techn. (ND2007) (Nice, France, 2007), p. 22.

[6] J. L. Tain and D. Cano-Ott, Nucl. Instrum. Methods Phys. Res. Sect. A 571, 728 (2007).

[7] J. L. Tain and D. Cano-Ott, Nucl. Instrum. Methods Phys. Res. Sect. A 571, 719 (2007).

[8] M. B. Gomez-Hornillos et al., Inter. Conf. on Nucl. Data for Sci. and Techn.-ND2010 (Jeju, Korea, 2010).

[9] H. Pentillä et al., Inter. Conf. on Nucl. Data for Sci. and Techn.-ND2010 (Jeju, Korea, 2010).

[10] B. Rubio, Inter. Conf. Frontiers of Nuclear Structure (Shanghai, China, 2006).

[11] C. Domingo-Pardo et al., Measurement of $\beta$-delayed neutrons around the third r-process peak (Proposal to GSIGPAC, 2010). 